\title{
Beyond Hemoglobin A1c: A Videographic Analysis of Conversations About Quality of Life and Treatment Burden During Clinical Encounters for Diabetes Care
}

\section{Shanzay Haider ( $\square$ shanzay.haider@yale.edu )}

Yale University School of Medicine https://orcid.org/0000-0001-9195-3517

Omar El Kawkgi

Mayo Clinic Minnesota

Jennifer Clark

Mayo Clinic Minnesota

Maggie Breslin

Mayo Clinic Minnesota

Kasey R. Boehmer

Mayo Clinic Minnesota

\section{Victor Montori}

Mayo Clinic Minnesota

Kasia J. Lipska

Yale School of Medicine: Yale University School of Medicine

\section{Research Article}

Keywords: quality of life, treatment burden, diabetes, clinician response

Posted Date: March 6th, 2021

DOI: https://doi.org/10.21203/rs.3.rs-282506/v1

License: (c) (1) This work is licensed under a Creative Commons Attribution 4.0 International License. Read Full License 


\section{Abstract}

Purpose: Diabetes care has largely focused on reducing the risk of complications by achieving hemoglobin A1c (HbA1c) targets; yet, whole-person care may be more effective and desirable. We sought to determine the nature of discussions about quality of life, burden of treatment, hypoglycemia, sexual function, and social support during diabetes-focused clinical encounters.

Methods: We analyzed 41 previously recorded clinical encounters with patients with type 2 diabetes from the control arms of practice-based trials of shared decision making. Two coders evaluated videos for discussions about aspects of life with diabetes: quality of life, burden of treatment, hypoglycemia, sexual function, and social supports. When an aspect was raised, coders evaluated the nature of the conversation, clinician responses, and time spent on discussing the aspect.

Results: Median length of the encounter was 15 minutes, 6 seconds (IQR: 11:16 - 20:23 minutes). Thirty five of 41 encounters (85.4\%), included some discussion of quality of life $(58.5 \%)$, burden of treatment $(51.2 \%)$, social support $(2.4 \%)$, or hypoglycemia $(9.8 \%)$. Sexual function was not discussed. On average, $4.5 \%(1.4 \%-5.5 \%)$ of the encounter time involved conversations about $\mathrm{HbA} 1 \mathrm{c}$ whereas $15.0 \%(0 \%-25 \%)$ of the encounter was spent on some aspect of quality of life, burden of treatment, social support, or hypoglycemia. If a topic related to quality of life was raised, clinicians most often responded by acknowledging patient's concern without providing a solution $(45.8 \%)$.

Conclusions: A significant part of the patient-clinician encounter involves discussion of quality of life and burden of treatment, but clinicians rarely address these issues by providing solutions.

\section{Introduction}

Diabetes mellitus is a common chronic condition, expected to affect as many as 366 million persons around the world by 2030 [1]. Glycemic control in patients with type 1 or type 2 diabetes can improve symptoms and may reduce the risk of long-term complications of the disease. Hemoglobin A1c (HbA1c) serves as a marker of medium-term glycemic control [8], and diabetes care has traditionally focused on targeting and maintaining specific levels of glycemic control (as measured by $\mathrm{HbA} 1 \mathrm{c}$ ) to reduce the risk of diabetic complications.

Yet, the challenges of living with diabetes, the setbacks and successes in improving glycemic control, and the value of the care received cannot be fully captured by changes in $\mathrm{HbA1c}$ levels.

Therapies to achieve target $\mathrm{HbA} 1 \mathrm{c}$ levels may impose significant burdens on patients (taking medications, injecting insulin, monitoring blood sugars, adjusting diet and exercise), may create or add to financial problems (increase premiums or out of pocket costs), may lead to hypoglycemia or other adverse events, may interact with other co-existing conditions and their treatments, and - overall - may not necessarily improve the quality of life, even in the long term $[1,2,3,4,5,12]$. 
Guidelines have tried to mitigate these potential harms by recommending individualized $\mathrm{HbA} 1 \mathrm{c}$ targets for patients who have complex medical and social conditions [20]. These recommendations, however, keep the attention of clinicians focused on glycemic control and not on the patient's situation as a whole. Clinicians caring for these patients may not fully appreciate their human - and often challenging circumstances. Understanding a patient's situation is necessary to co-create care plans that fit well within that individual's life. This requires care to be safe, effective, and desirable while addressing the complexity of the patient's biology, priorities, and feasibility given the complexity of his/her life.

The purpose of this study was to determine how frequently topics related to quality of life, treatment burden, social supports, sexual function, and hypoglycemia are raised during routine clinical encounters and how they are raised. Additionally, we observed clinician response to these issues. We took advantage of video-recorded encounters obtained during clinical trials of shared decision-making tools using the control group that did not receive the intervention to represent usual care. We quantified how often specific topics were raised and quantified the time of these discussions. Based on a previous study that examined diabetes-specific quality of life and burden of treatment, we hypothesized that at least some of these topics were routinely brought up in discussions by patients [6].

However, given the focus of the current guidelines and traditional HbA1c-directed approach to diabetes care, we hypothesized that clinicians would have a low level of comfort addressing aspects related to the patient situation, thus spend less time discussing these topics when compared to HbA1c.

\section{Methods}

All procedures were approved by the Mayo Clinic IRB (\#19-004560).

\section{Data Sources}

We conducted a videographic analysis of previously recorded clinical encounters with patients living with type 2 diabetes from past clinical trials [6, 9]. Eligible video-recordings captured clinical encounters as part of practice-based randomized trials comparing care-as-usual to care with a shared decision-making (SDM) tool.

\section{Study Population}

Seventy-one video recordings were available and reviewed for inclusion within the study analysis. These encounters involved a clinician caring for an adult (age $\geq 18$ ) with type 2 diabetes mellitus with an $\mathrm{HbA} 1 \mathrm{c}>7 \%$ and not using insulin.

Videos were excluded if they included the intervention arm of a shared decision-making tool for diabetes medications because these tools then guided conversation between the clinician and patient; however, videos were included within the analysis if a decision aid for another condition was used.

\section{Data Analysis}


Two coders $(\mathrm{OK}, \mathrm{SH})$ working independently reviewed each video recording after ensuring they could agree on their codes $\geq 90 \%$ of the time, which approximated 3 videos analyzed by both coders to achieve agreement. During this calibration process, a codebook was created that defined aspects of quality of life, burden of treatment, hypoglycemia, sexual function, and social support that were subsequently coded. The domains coded were determined a priori based on existing literature and surveys of patients with type 2 diabetes that focused on each of these domains $[2,3,4,6,7,12]$. The details of each domain were then determined inductively as coders discussed video content. Coders would discuss the nature of the situation and determine under which circumstances the situation should be coded as pertaining to quality of life, burden of treatment, social supports, sexual function, or hypoglycemia. When an aspect was noted, reviewers considered the questions listed in Appendix 2 to further characterize the situation, to assess the time spent on the discussion of each of these aspects, the time spent discussing HbA1c, and the total encounter duration. Once calibration was established, coders individually evaluated the rest of the videos included in the final analysis with discussion and clarification, as needed. As videos were analyzed, the codebook was updated after discussion to reflect emerging situations that arose during video analysis. Descriptive analyses were used to summarize findings.

\section{Results}

Of the 71 available video recordings, 41 met eligibility criteria and included in our analysis. Among clinicians, majority were family medicine practitioners followed by internal medicine practitioners. The median length of the encounter was 15 minutes, 6 seconds (IQR: 11:17 - 20:23 minutes). Most encounters, 35 of $41(85.4 \%)$, included some discussion of quality of life, burden of treatment, social support, or hypoglycemia (Table 1).

\section{Aspects of the patient situation raised during clinical encounters}

Patients most often raised concerns regarding their quality of life ( $58.5 \%$ of encounters). Most often, these conversations focused on pain or diabetes-related stress. Of these quality of life discussions, $62.5 \%$ were raised by the patient. Burden of treatment was also discussed frequently, during 21 (51.2\%) of the studied encounters. Aspects of burden of treatment that were discussed included difficulties with implementing healthy diet and exercise routines (61.9\%), challenges with administering and/or taking medications (47.6\%). Hypoglycemia was only discussed in 4 encounters (9.8\%); the discussion focused primarily on the frequency of hypoglycemia. Social support surfaced in only 1 encounter $(2.4 \%)$ in the context of discussing the patient's relationship with their family. Sexual function was not discussed during any of the encounters.

Time spent discussing quality of life, burden of treatment, hypoglycemia, and social support

Table 1 demonstrates that, on average, $4.5 \%$ of the encounter time involved conversations about $\mathrm{HbA} 1 \mathrm{c}$ levels whereas $15.0 \%$ of the encounter time was spent on some aspect of quality of life, burden of treatment, hypoglycemia, or social support. 
In response to quality of life concerns, clinicians reacted by asking elaborating questions in 21 out of the 24 encounters. The most common type of response (11 of the 24 encounters) was to acknowledge the patient's concern but not propose a solution. Regarding aspects pertaining to burden of treatment, clinicians acknowledged the problem (38\%), suggested a period of observation and monitoring (57\%), and offered a direct solution, such as a change in the treatment program (38\%). Clinicians responded to issues of hypoglycemia by proposing a period of monitoring in all 4 of the encounters in which hypoglycemia was raised (100\%).

How these topics affected the flow of the encounter differed among clinicians and visits. We found instances where the patient's concern led to a collaborative discussion between clinician and patient, and other times where it was disruptive to the flow of the encounter. For example, in one encounter, a physician addressed the patient's overall wellbeing, which led to a discussion about the patient's chronic abdominal pain. The physician acknowledged the patient's concerns by asking elaborating questions and inquiring about what workup has been done so far. Throughout this encounter lasting 12 minutes, the physician and patient took turns leading the conversation which revolved around patient concerns. In contrast, the conversation in another encounter lasting 13 minutes was directed by the clinician and focused solely on clinician's concerns about lab work. In this encounter, the patient brought up concerns about her back pain, but after quickly acknowledging the patient's pain, the clinician immediately redirected the conversation back to lab work. In both example encounters, the physician's response was to acknowledge the patient's concern, however, the differences in navigating the concerns were apparent in how the encounter flowed and how it was directed.

\section{Discussion}

In our study, patients with diabetes and clinicians commonly discussed quality of life and the cumulative burden of illness associated with diabetes and other comorbidities. On average, discussions about quality of life, burden of treatment, hypoglycemia, and social supports took up twice as much time as discussions focused on controlling $\mathrm{HbA} 1 \mathrm{c}$ levels. Clinicians recognized and routinely acknowledged patient concerns. However, except for treatment burden, clinicians rarely offered concrete modifications that would mitigate problems identified in the care plan.

There have been numerous studies and reviews assessing burden of treatment and quality of life in patients with diabetes. While a significant portion of the literature is consistent with our results, there were some studies with conflicting findings. In one study, patients with diabetes at target glycemic goal expressed poorer quality of life and increased treatment burden compared to control subjects without diabetes [12]. In contrast, other studies have found similar reported quality of life in type 2 diabetes patients who were at $\mathrm{HbA} 1 \mathrm{c}$ goal when compared to non-diabetic controls $[16,17]$. The majority of patients included in these studies were on minimal diabetic regimens and followed mostly in the primary care setting. Few studies assessed clinician's response to aspects of quality of life and treatment burden 
during clinical encounters $[12,16,17]$. In contrast, our analysis captured both the nature of discussion around quality of life as well as clinician responses to concerns raised. Assessing the clinician's response - and documenting the lack of clinician's response - reveals potential gaps in care which could be addressed with resources and training to better serve patient needs during a clinical encounter.

The nature of discussions about quality of life and treatment burden in our study are largely consistent with prior literature. Prior literature has repeatedly highlighted the challenges in medication management and lifestyle changes in patients with diabetes, with most studies using patient surveys [21]. In studies where patient interviews were conducted, specifically about treatment burden, the most common issues were cost of treatment, followed by problems with taking medications, and activity limitations $[21,22,23]$. Yet again, this literature lacks insight into how these topics arise in clinical encounters and how clinicians respond when aspects of treatment burden arise.

Clinicians are often aware of the burden patients with diabetes encounter, yet it is often unaddressed [13]. This is despite the fact that personal stressors can affect glucose levels and patient's wellbeing [14]. In one study examining treatment burden in patients with diabetes, the prevalence of treatment burden was high but did not result in any concrete solutions for the patients [6].

Although our findings indicate that clinicians' and patients' attention is often directed well beyond just $\mathrm{HbA1c}$ levels, clinicians infrequently address these quality of life issues directly. There are several potential reasons for this. First, the way these issues are raised may not result in a productive discussion to develop solutions. For example, some clinicians may acknowledge issues but see them as falling outside of the scope of their practice, or as interruptions to their medical agenda. Second, clinicians may lack the skills and/or tools to respond to issues raised. Third, the burden of treatment may be accepted as "normal" by clinicians.

In our study, issues related to sexual function did not come up in any of the encounters. Diabetes is a known risk factor for sexual dysfunction: men with diabetes experience a threefold increase in rates of sexual dysfunction compared to men without diabetes [7]. Sexual dysfunction has also been described in women with diabetes at increased frequency compared with women without diabetes although limited literature is available on this topic [11]. Given its importance to quality of life and cardiovascular prognosis [7], this aspect needs further study. It is possible that patients and clinicians may be uncomfortable discussing sexual function or may not consider it necessary to address.

Our results suggest the need for an intervention to promote productive discussions about quality of life, treatment burden, sexual function, social supports, and hypoglycemia to improve patient-centered care. Our group has begun to develop an intervention that would help patients set an agenda for the visit with regards to important aspects of Quality of life, Burden of treatment, Social integration, and Avoidance of Future Events-abbreviated as QBSAFE approach. This intervention, aimed at improving the clinicianpatient encounter, is undergoing feasibility and acceptability testing funded by a grant from the National Institutes of Aging. 
There are several limitations to this study. Our videos captured a single visit, and some did not capture the whole visit as they were stopped prior to finishing the encounter (a prerogative of trial participants). It is possible that discussions focused on $\mathrm{HbA} 1 \mathrm{c}$ and other aspects of diabetes may have already taken place in past and/or future visits. No videos included patients on insulin, even though insulin may be particularly burdensome to patients. Discussions pertaining to hypoglycemia, burden of treatment, and quality of life may be even more common during encounters with patients receiving insulin. While these are limitations of the secondary analysis of existing recordings, this study is made stronger by using all available and eligible recordings, evaluation by independent coders, and the triangulation of our findings with existing literature.

In conclusion, our analysis found a significant proportion of patient-clinician encounters involved discussions surrounding quality of life and treatment burden. Topics like social support, and hypoglycemia were discussed less frequently, while sexual function was not discussed at all. Overall, the encounter time devoted to quality of life and treatment burden far exceeded time spent discussing $\mathrm{HbA} 1 \mathrm{c}$. However, these discussions about aspects of life with diabetes rarely resulted in changes in the care program. Accordingly, we propose to develop an intervention to change conversations between patients and clinicians so that they can co-develop plans that respond to challenges faced by patients. Such a patient-centered approach may help improve patient-clinician partnerships, care processes and health outcomes.

\section{Declarations}

Funding: This study was funded by a National Institute on Aging R21 grant (1R21AG061427). The funding body did not have a role in the study design and will not take part in data collection, analysis, or interpretation for the study. Funding body did not participate in writing this manuscript.

Conflicts of interest/Competing interests: $\mathrm{KJL}$ reports that she receives support from the Centers for Medicare \& Medicaid Services (CMS) to develop and evaluate publicly reported quality measures. Other authors declare that they have no competing interests.

Availability of data and material: Videos for analysis were obtained from prior studies and approved for use by Mayo Clinic IRB.

\section{Authors contributions:}

Conception and design of the work: all authors

Acquisition of data: VM

Data analysis: $\mathrm{SH}$ and OEK

Interpretation of data for the work: all authors 
Drafting of the manuscript: SH

Revision for critically important intellectual content: all authors

Final approval of the version to be published: all authors

Funding and supervision were provided by VM and KJL.

Ethics approval: All procedures were approved by the Mayo Clinic IRB under protocols: (\#19-004560), (\#09-008640), and (\#10-006952).

Consent to participate: Informed consent was obtained prior to interview and video recording.

Consent for publication: Participants signed informed consent about publication of data.

\section{References}

1. Wild, S., Roglic, G., Green, A., Sicree, R., \& King, H. (2004, May 01). Global Prevalence of Diabetes. Retrieved June 25, 2020, from https://care.diabetesjournals.org/content/27/5/1047

2. Trikkalinou A, Papazafiropoulou AK, Melidonis A. Type 2 diabetes and quality of life. World $J$ Diabetes. 2017;8(4):120-129. doi:10.4239/wjd.v8.i4.120

3. K W Watkins, C M Connell, J T Fitzgerald, L Klem, T Hickey, B Ingersoll-Dayton. Effects of adults' selfregulation of diabetes on quality of life outcomes. Diabetes Care Oct 2000, 23 (10) 1511-1515; DOI: 10.2337/diacare.23.10.1511

4. Ngo-Metzger, Q., Sorkin, D.H., Billimek, J. et al. The Effects of Financial Pressures on Adherence and Glucose Control Among Racial/Ethnically Diverse Patients with Diabetes. J GEN INTERN MED 27, 432-437 (2012). https://doi.org/10.1007/s11606-011-1910-7

5. Parikh, P.B., Yang, J., Leigh, S. et al. The Impact of Financial Barriers on Access to Care, Quality of Care and Vascular Morbidity Among Patients with Diabetes and Coronary Heart Disease. J GEN INTERN MED 29, 76-81 (2014). https://doi.org/10.1007/s11606-013-2635-6

6. Krista Bohlen, Elizabeth Scoville, Nathan D. Shippee, Carl R. May, Victor M.Montori. Overwhelmed Patients. Diabetes Care Jan 2012, 35 (1) 47-49; DOI: 10.2337/dc11-1082

7. Maiorino, M., Bellastella, G., \& Esposito, K. (2014). Diabetes and sexual dysfunction: Current perspectives. Diabetes, Metabolic Syndrome, and Obesity: Targets and Therapy.

8. UKPDS Group. UK Prospective Diabetes Study 17: a nine-year update of a randomized, controlled trial on the effect of improved metabolic control on complications in non-insulin-dependent diabetes mellitus. Ann Intern Med 1996;124:136-45

9. Mullan RJ, Montori VM, Shah ND, et al. The Diabetes Mellitus Medication Choice Decision Aid: A Randomized Trial. Arch Intern Med. 2009;169(17):1560-1568. doi:10.1001/archinternmed.2009.293

10. Maiorino MI, Bellastella G, Esposito K. Diabetes and sexual dysfunction: current perspectives. Diabetes Metab Syndr Obes. 2014;7:95-105. Published 2014 Mar 6. doi:10.2147/DMSO.S36455 
11. Enzlin P, Rosen R, Wiegel M, et al. Sexual dysfunction in women with type 1 diabetes: long-term findings from the DCCT/ EDIC study cohort. Diabetes Care. 2009;32(5):780-785. doi:10.2337/dc081164

12. Fisher L, Polonsky WH, Hessler D. Addressing diabetes distress in clinical care: a practical guide. Diabetic Medicine. 2019; 36: 803-812. DOI: 10.1111/dme.13967

13. Fisher L, Mullan JT, Skaff MM, Glasgow RE, Arean P, Hessler D. Predicting diabetes distress in patients with Type 2 diabetes: a longitudinal study. Diabetic Medicine. 26: 622-627. doi:10.1111/j.1464-5491.2009.02730.x

14. Albright TL, Parchman M, Burge SK. Predictors of self-care behavior in adults with type 2 diabetes: an RRNeST study. Fam Med2001; 33: 354- 360.

15. Lau CY, Qureshi AK, Scott SG. Association between glycemic control and quality of life in diabetes mellitus. Journal of Postgrad Med. 2004; 50(3): 189-193.

16. Fisher L. Quality of Life: Issues of Conceptualization and Measurement. New Orleans, LA: American Diabetes Association, 2016.

17. Perrin N, Davies M, Robertson N, Snoek FJ, Khunti K. The prevalence of diabetes-specific emotional distress in people with Type 2 diabetes: a systematic review and meta-analysis. Diabet Med2017; 34: 1508- 1520.

18. Kasteleyn MJ, de Vries L, van Puffelen AL, Schellevis FG, Rijken M, Vos RC et al . Diabetes-related distress over the course of illness: results from the Diacourse study. Diabet Med2015; 32: 16171624.

19. Snoek FJ, Bremmer MA, Hermanns N. Constructs of depression and distress in diabetes: time for an appraisal. Lancet Diabetes Endocrino/2015; 858: 135- 137.

20. Qaseem A, et al. Hemoglobin A1c targets for glycemic control with pharmacologic therapy for nonpregnant adults with type 2 diabetes mellitus: a guidance statement update from the American College of Physicians. Annals of Internal Medicine. 2018.

21. Tran, VT., Barnes, C., Montori, V.M. et al.Taxonomy of the burden of treatment: a multi-country webbased qualitative study of patients with chronic conditions. BMC Med13, 115 (2015). https://doi.org/10.1186/s12916-015-0356-x

22. Sav A, Kendall E, McMillan SS, Kelly F, Whitty JA, King MA, Wheeler AJ. 'You say treatment, I say hard work': treatment burden among people with chronic illness and their careers in Australia. Health Soc Care Community. 2013 Nov;21(6):665-74. doi: 10.1111/hsc.12052. Epub 2013 May 23. PMID: 23701664.

23. Eton DT, Ramalho de Oliveira D, Egginton JS, Ridgeway JL, Odell L, May CR, Montori VM. Building a measurement framework of burden of treatment in complex patients with chronic conditions: a qualitative study. Patient Relat Outcome Meas. 2012;3:39-49. doi: 10.2147/PROM.S34681. Epub 2012 Aug 24. PMID: 23185121; PMCID: PMC3506008.

24. Centers for Disease Control and Prevention. Measuring healthy days: population assessment of health-related quality of life. Centers for Disease Control and Prevention, Atlanta, Georgia 2000. 
25. Gallacher, K.I., May, C.R., Langhorne, P. et al.A conceptual model of treatment burden and patient capacity in stroke. BMC Fam Pract19, 9 (2018). https://doi.org/10.1186/s12875-017-0691-4

\section{Appendix}

Appendix 1. QBSAFE Videographic Codebook: The purpose of this codebook is to help identify aspects and examples of quality of life, burden of treatment, social support, hypoglycemia, and sexual dysfunction for the use of coders analyzing clinician video encounters. 
health-related Quality of Life [QOL]

"An individual's or group's perceived physical and mental health over time...includes physical and mental health perceptions and their correlates..." [1]. overall general health

physical well being

mental health i.e. mood, depression, anxiety, stress, etc.

pain

- code as QOL if diet/exercise limited by pain

○ numbness/tingling

fatigue

- code as QOL if diet/exercise limited by fatigue

- visual impairment

- difficulty in carrying out daily activities due to disability i.e. weakness from prior stroke, amputation, etc.

$>$ mention of other co-morbid condition affecting any of the above aspects

Burden of Treatment [BoT]

"The workload of healthcare experienced by those with long-term conditions and the impact it has on well-being" [2]. difficulties in administering and/or taking meds

- difficulties in frequency of drug administration

trouble with self-monitoring

problems with attending or keeping doctor appointments

issues with transportation to and from appointments

- problems with scheduling doctor follow ups

- financial burden of disease

- difficulties in relationships secondary to BoT 
Hypoglycemia

Defined as an episode of blood glucose reading less than 70 .

$>$ Did not include episodes where patients felt

hypoglycemic but had normal blood glucose levels.

$>$ Did not include episodes where patients described

symptoms and did not check blood glucose levels. reduced awareness of hypoglycemia

- frequency of hypoglycemia severity of hypoglycemia

Social support

Includes both objective absence of social contact as well as perceived loneliness. feeling of loneliness

lack of social network

- difficulty connecting with social network/circle

reports issues in spending time with friends and family not otherwise related to BoT

Sexual Dysfunction

Any report of sexual dysfunction in any cohort of patients. erectile dysfunction

decreased libido

other issues related to sexual dysfunction

\section{Appendix 2. Video analysis questions}




\section{If an aspect of quality of life, burden of treatment, hypoglycemia, social support was brought up during the clinical scenario, the following questions were used to analyze the scenario:}

What aspect was brought up? [i.e. QOL, BoT, Hypoglycemia]

Who initiated the conversation?

What was the nature of the concern? [refer to table 1.]

When conversation was initiated, did the clinician address the problem?

[This was defined as the clinician asking any follow up questions or further discussion of the topic]

If the clinician addressed the problem, how did they go about addressing it:

Acknowledge without proposing a solution

Propose to monitor and/or observe

Order additional testing

Referral to specialist

Change of medication regiment or treatment

[including addition of new medication or diet/exercise recommendations]

Change of frequency or method of monitoring

Change in frequency of follow up

Time spent on each aspect brought up

\section{Tables}

\section{Table 1. Results}




\section{Overall}

Video-recorded encounters analyzed

Encounters with any aspect of QBSAFE raised

Encounter duration, median (IQR), minutes:seconds

Time spent on HbA1c, median (IQR), minutes:seconds

Average percentage of encounter time spent on $\mathrm{HbA1c}$ (SD)

Average percentage of encounter time spent on QBSAFE (SD)

\section{Quality of Life (QOL)}

Encounters where a QOL topic was brought up

Encounters with $\geq 1$ QOL topic

Patient brought up QOL topic

Nature of QOL topic:

Overall health/ general health

Physical well being

Mental health

Fatigue

Pain

Sexual function

Clinician responded to QOL topic
$N(\%)$

41

$35(85.4 \%)$

15:06 (11:17, 20:23)

$0: 33(0: 13-0: 50)$

$4.5 \%$

$(1.4 \%-5.5 \%)$

$15.0 \%$

(0\%-25\%) $\mathrm{n} / \mathrm{N}(\%)$

$24 / 41(58.5 \%)$

8/41 (19.5\%)

$15 / 24(62.4 \%)$

Type of clinician response to QOL topic (36 actions taken by clinician in response to topic)

Acknowledged without proposing a solution $11 / 24(45.8 \%)$

Proposed to gather more information (observe, monitor, obtain more tests, refer to specialist)

$4 / 24(16.7 \%)$

$3 / 24(12.5 \%)$

$4 / 24(16.7 \%)$

$2 / 24(8.3 \%)$

$16 / 24(66.7 \%)$

$0 / 24(0 \%)$

$21 / 24(87.5 \%)$

Change of medication regimen or treatment

Change of frequency or method of monitoring

Change in frequency of follow up

$12 / 24(50 \%)$

$7 / 24(29.2 \%)$

$0 / 24(0 \%)$

$0 / 24(0 \%)$

Time spent discussing QOL topic, median (IQR), (minutes:seconds) 
Burden of Treatment (BoT)

Encounters were a BoT topic was brought up

Encounters with $\geq 1$ BoT topic

Patient brought up BoT topic

Nature of BoT topic:

Difficulties in administering and/or taking meds

Difficulties in frequency of drug administration

Trouble with self-monitoring

Problem with attending or keeping doctor appointments

Financial burden of disease

Difficulties in relationships due to BoT

Difficulties in adherence to diet and exercise

Clinician responded to BoT topic

Type of clinician response to BoT topic

Acknowledged without proposing a solution

Proposed to gather more information (observe, monitor, obtain more tests, refer to specialist)

Change of medication regimen or treatment

Change of frequency or method of monitoring

Change in frequency of follow up

Time spent discussing BoT topic, median (IQR), (minutes:seconds)
$8 / 21(38 \%)$

$\mathrm{n} / \mathrm{N}(\%)$

$21 / 41(51.2 \%)$

$8 / 41(19.5 \%)$

$9 / 21(43 \%)$

$10 / 21(47.6 \%)$

$0 / 21(0 \%)$

$1 / 21(4.8 \%)$

$0 / 21(0 \%)$

$3 / 21(14.3 \%)$

$0 / 21(0 \%)$

$13 / 21(61.9 \%)$

$19 / 21(90.4 \%)$

$12 / 21(57 \%)$

$8 / 21(38 \%)$

$1 / 21(5 \%)$

$1 / 21(5 \%)$

$1: 30$

$(0: 45$ - 3:03)

Hypoglycemia

$\mathrm{n} / \mathrm{N}(\%)$

Encounters were hypoglycemia was brought up

$4 / 41(9.8 \%)$

Patient brought up hypoglycemia

$3 / 4(75 \%)$

Nature of discussion about hypoglycemia: 
Reduced awareness of hypoglycemia

$1 / 4(25 \%)$

Frequency of hypoglycemia

$3 / 4(75 \%)$

Severity of hypoglycemia

$0 / 4(0 \%)$

Clinician responded to hypoglycemia

$4 / 4(100 \%)$

Type of clinician response to hypoglycemia ( 5 actions taken by clinician in response to topic)

Acknowledged but no solution proposed

$0 / 4(0 \%)$

Proposed to monitor or observe

$4 / 4(100 \%)$

Time spent discussing hypoglycemia, median, (minutes:seconds)

$0: 32$

\section{Social Support}

Encounters were social support was brought up

Patient brought up social support

Nature of discussion about social support:

Loneliness

Time with friends and family

Clinician responded to social support topic

Type of clinician response to social support

Proposed to monitor or observe

Time spent discussing social support, median, (minutes:seconds) $\mathrm{n} / \mathrm{N}(\%)$

$1 / 41(2.4 \%)$

$0 / 1(0 \%)$

$0 / 1$

1/1 (100\%)

1/1 (100\%)
1/1 (100\%)

0:23 